\title{
Identificación de áreas de riesgo agroclimático para el sistema de ganadería de leche en el Valle de Ubaté y Chiquinquirá y Alto Chicamocha
}

\section{Identification of Agroclimatic risk areas for dairy livestock systems in Valle de Ubaté y Chiquinquirá and Alto Chicamocha}

\section{FABIO ERNESTO MARTÍNEZ ${ }^{1,3}$ \\ LILIANA CASTILLO V. ${ }^{1}$ \\ EDWIN ROJAS B. ${ }^{1}$ \\ DOUGLAS A. GÓMEZ-LATORRE' \\ ELIZABETH AGUILERA G. ${ }^{2}$}

Valle de Ubaté y Chiquinquirá, Colombia.

Foto: D.A. Gómez-Latorre

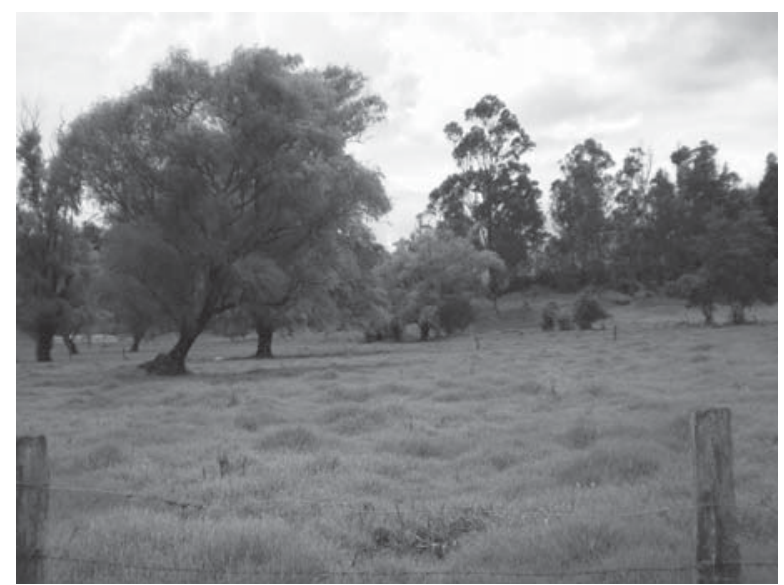

\section{RESUMEN}

En este estudio se determinó la aptitud agroclimática para el sistema ganadero de leche mediante la formulación del "Índice de Aptitud Agroclimática (IAC)". El índice se construyó a partir del análisis territorial de variables limitantes como la pendiente, la exposición a heladas, el índice de humedad y temperatura para Bos taurus, la frecuencia de ocurrencia de condiciones de déficit hídrico en el suelo calculado mediante balances hídricos, el número de días sin lluvia y la precipitación media acumulada. El índice agroclimático permitió identificar áreas críticas para el sistema de ganadería en los periodos secos de diciembre a febrero, como primer trimestre seco y de junio a agosto como segundo trimestre seco. Los resultados mostraron que en el primer trimestre seco hay una mayor área con limitaciones agroclimáticas en comparación con el segundo trimestre seco; cerca de 192.369 ha ubicadas en la región del Alto Chicamocha presentaron los valores más bajos del IAC, indicando una menor aptitud agroclimática: Sogamoso, Corrales, Tutazá, Belén, Santa Rosa de Viterbo, Floresta, Nobsa, Sotaquirá, Cerinza, Pesca, Chíquiza, Tausa, Samacá, Cucaita, Sogamoso y Firavitoba fueron los municipios con más baja aptitud agroclimática para los dos periodos secos, tanto en términos de las limitantes evaluadas como del IAC. Las áreas con menores limitaciones (con valores más altos del IAC) se observaron en el $12 \%$ del área estudiada y correspondieron principalmente a los municipios de Oicatá y Cuitiva en la región del Alto Chicamocha, y Cucunubá en el valle de Ubaté y Chiquinquirá.

\footnotetext{
Centro de Investigación Tibaitatá, Corporación Colombiana de Investigación Agropecuaria (Corpoica), Mosquera (Cundinamarca, Colombia).

2 Consultor independiente, Bogotá (Colombia).

3 Autor para correspondencia. femartinez@corpoica.org.co
} 
Palabras clave adicionales: agroclimatología, eventos extremos, producción de leche, variabilidad climática.

\section{ABSTRACT}

In this study, the agroclimatic suitability for dairy livestock systems was determined with an "Agroclimatic suitability index (ASI)". The index was constructed from the territorial analysis of restrictive variables such as slope, frost exposure, Bos taurus temperature and humidity index. The soil water deficit frequency was calculated with water balances, days without rain and accumulated average precipitation. The agroclimatic index identified critical areas for dairy livestock systems in the main dry periods (December to February and June to August). The results showed that in the first dry quarter, there was a larger area with agroclimatic limitations as compared to the second dry quarter. About 192,369 ha, located mainly in the Alto Chicamocha, had the lowest IAC values, indicating a lower agroclimatic suitability. Sogamoso, Corrales, Tutaza, Belen, Santa Rosa de Viterbo, Floresta, Nobsa, Sotaquira, Cerinza, Pesca, Chiquiza, Tausa, Samaca, Cucaita, Sogamoso and Firavitoba were the municipalities with the lowest agroclimatic suitability index for the two dry periods. The areas with the lowest limitations (with higher IAC values) were observed in $12 \%$ of the studied area and corresponded mainly to the municipalities of Oicata and Cuitiva in the Alto Chicamocha and Cucunuba in the Valle de Ubate and Chiquinquira.

Additional key words: agroclimatology, climatic variability, extreme events, milk production.

Fecha de recepción: 08-02-2017 Aprobado para publicación: 15-04-2017

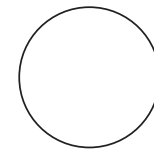

La variabilidad climática de la lluvia y la temperatura ha sido el principal factor de estacionalidad y variabilidad en la producción agropecuaria en los países en desarrollo, constituyendo uno de los principales factores de la falta de seguridad alimentaria. Anomalías en las lluvias y la temperatura del aire, sequías, heladas, inundaciones y otros eventos climáticos extremos asociados con la variabilidad climática han afectado históricamente áreas dedicadas a actividades agrícolas y pecuarias, reduciendo la productividad, generando pérdidas económicas y en general, afectando la sostenibilidad de los sistemas productivos agropecuarios (FAO, 1997).

Tales eventos, en conjunción con otros factores ambientales, tecnológicos y socioeconómicos determinan la vulnerabilidad de los sistemas de producción agropecuaria frente al cambio y la variabilidad climática, generalmente mayor para los campesinos y comunidades rurales más pobres (Sivakumar et al., 2006).

Diversos estudios han analizado los impactos del cambio y la variabilidad climática sobre la agricultura, y predicen altas probabilidades de que estos impactos se acentúen en el futuro (CEPAL 2015), además muestran que existe una relación inversa entre los fenómenos climáticos extremos y los rendimientos agropecuarios, debido en parte al creciente aumento de procesos de desertificación, degradación de tierras y fuentes hídricas (IPCC, 2012).

En Colombia, aproximadamente 30 millones de hectáreas están dedicadas a actividades pecuarias, de las que dependen alrededor de 400.000 ganaderos que producen diariamente al menos 15 millones de litros de leche (DANE, 2016). El 40\% de esta producción se concentra en los altiplanos cundiboyacense, nariñense y antioqueño, principalmente en pequeños predios de economía campesina. La producción presenta ciclos de alta producción en temporadas de lluvia y de baja producción en temporadas de bajas precipitaciones. Estos ciclos están determinados principalmente por los volúmenes de lluvias que definen la disponibilidad de alimentación para los animales (Arreaza, 2012; Arreaza et al., 2012; Sánchez et al., 2009; Cardozo et al., 2008).

Factores territoriales, como la altitud, pendiente, topografía y vegetación, determinan la ocurrencia e 
impacto de fenómenos extremos en conjunción con manifestaciones locales de la variabilidad climática, que en el altiplano cundiboyacense dependen principalmente del movimiento estacional de la Zona de Convergencia Intertropical ZCIT y su interacción con los eventos ENSO (fases El Niño y La Niña) (Gómez-Latorre, 2014). Durante los últimos fenómenos El Niño y La Niña, en los cuales se presentaron fuertes sequías y heladas en 2010, 2015 y 2016, así como excesos de lluvias y las más intensas inundaciones en 2011 y 2012; el sector ganadero acumuló pérdidas por más de 4,3 billones de pesos, que ocasionaron la muerte o desplazamiento de más de 343.000 animales entre finales de 2009 y 2012. Estas pérdidas han influido en la disminución del inventario bovino, que desde 2010, se ha reducido en cerca del 4\% (Tapasco et al., 2015). Sin embargo, debido a sus características ambientales y las cualidades de sus hatos ganaderos, el altiplano cundiboyacense es la mejor zona del país para la producción de leche, tanto en términos de calidad como de cantidad (Holmann et al., 2004).

Cerca de 150.000 ha en las regiones lecheras del altiplano Cundiboyacense fueron afectadas durante el fenómeno de El Niño de 2009-2010 y la subsesecuente ocurrencia de La Niña de 2010-2012. Reducciones de hasta 82,5 millones de litros de leche se presentaron en diciembre de 2009 debido deficiencias hídricas, inundaciones y anegamientos; de la misma forma, la reducción de 63,5 millones de litros registrada durante febrero de 2010 ocurrió gracias a la presencia de heladas (ANALAC, 2012), fenómeno que en el altiplano cundiboyacense tiene una probabilidad de ocurrencia cercana al $90 \%$ en los meses de diciembre a febrero especialmente (Mayorga et al., 2008).

La zonificación de la aptitud agroclimática en el territorio constituye una estrategia de manejo del riesgo que orienta la implementación de medidas preventivas y de adaptación frente a las adversidades asociadas con la variabilidad climática (Brunini et al., 2010). Actualmente existen diversas aproximaciones o técnicas para la zonificación de la aptitud agroclimática de un espacio geográfico para diferentes cultivos. Particularmente la construcción de índices es una herramienta clave para la determinación del potencial agrícola y permite simplificar el análisis territorial del impacto de eventos de variabilidad sobre sistemas de producción específicos. Éstos índices pueden incluir la evaluación de riesgo climático asociado a heladas, déficit y exceso hídrico, sequía y altas temperaturas en escenarios de variaciones temporales y variabilidad climática (Brunini et al., 2010; Moeletsi y Walker,
2012). Un ejemplo de ello es el Poone Agro Climatic Suitability Index (PACSI), generado para integrar los riesgos climáticos más importantes para la producción de maíz en Free State, Sudáfrica. El índice se construyó a partir del análisis de las temporadas de lluvias, el riesgo a heladas en el periodo de crecimiento y el riesgo de sequía (WRSI) calculado a partir del requerimiento de agua en el periodo de crecimiento del maíz.

De la misma forma, Brunini et al. (2009) y Yazdanpanah et al. (2001) consideran que la zonificación de la aptitud agroclimática es una herramienta clave para la determinación del potencial agrícola, la planificación territorial y el manejo de los suelos en áreas de producción agropecuaria. Por lo anterior, el objetivo de este estudio fue identificar áreas de riesgo agroclimático para el sistema de ganadería de leche en los valles de Ubaté y Chiquinquirá y del Alto Chicamocha, mediante el análisis de la aptitud agroclimática durante los periodos secos de diciembre a febrero (DEF) y de junio a agosto (JJA). Los resultados constituyen una base para el ordenamiento productivo agropecuario y permitirán orientar futuras investigaciones que vinculen la influencia de factores meteorológicos en la productividad y el bienestar animal, así como la planificación de actividades de manejo para reducir la vulnerabilidad del sistema asociada frente al cambio y la variabilidad climática.

\section{MATERIALES Y MÉTODOS}

\section{Área de estudio}

El valle de Ubaté y Chiquinquirá, que comprende los municipios de: Tausa, Sutatausa, Carmen de Carupa, Cucunubá, Ubaté, Lenguazaque, Guachetá, Fúquene, Susa, Guasca, Villapinzón y Simijaca en Cundinamarca y San Miguel de Sema, Saboya, Caldas, Ráquira y Chiquinquirá en Boyacá, tiene una extensión aproximada de 220.300 ha y está ubicado entre los $5^{\circ} 03^{\prime}$ y $5^{\circ} 54^{\prime} \mathrm{N}$ y $73^{\circ} 21$, y $72^{\circ} 30$, W. La altitud varía entre los 2.500 y 3.000 msnm y su precipitación es cercana a los $1.000 \mathrm{~mm}$ al año (Corpoica, 2004).

El valle del Alto Chicamocha, que comprende los municipios de: Samacá. Cucaita, Tunja, Sáchica, Chíquiza, Motavita, Combita, Oicatá, Sotaquirá, Tuta, Toca, Pesca, Tota, Cuítiva, Duitama, Firavitoba, Iza, Nobsa, Paipa, Santa Rosa de Viterbo, Sogamoso, Tibasosa, Corrales, Busbanzá, Floresta, Cerinza, Belén y 
Tutazá, está ubicado entre los $4^{\circ} 33^{\prime}$ y $5^{\circ} 8^{\prime} \mathrm{N}$ y $73^{\circ} 35^{\prime}$ y $72^{\circ} 51^{\prime}$ W, tiene una extensión de 582.572 ha y su altitud varía entre los 2.000 y $3.000 \mathrm{msnm}$, con precipitaciones anuales entre 500 y $1.000 \mathrm{~mm}$ (Fig. 1) (Corpoica, 2004).

En ambos valles predominan los paisajes de montaña, altiplanicie, planicie y valle (IGAC, 2000; IGAC, 2005) y el comportamiento de las lluvias principalmente es de carácter bimodal, con periodos de lluvias de marzo a mayo en el primer semestre y de septiembre a noviembre en el segundo (Gómez-Latorre, 2014).

\section{Aproximación}

La zonificación de la aptitud agroclimática se realizó teniendo en cuenta la definición del Panel Intergubernamental de Expertos sobre el Cambio Climático
(IPCC, 2012), que se basa en la exposición del sistema a eventos climáticos extremos, definida por su ubicación en el territorio. La metodología se basó en la construcción de mapas temáticos de las principales limitantes territoriales, climáticas y agroclimáticas para el sistema ganadero de leche. El análisis de la aptitud se determinó mediante el cálculo del Índice de Aptitud Agroclimática (IAC) obtenido a través de un análisis espacial multicriterio (Díaz y López, 2000) mediante una ponderación de los factores limitantes para el sistema productivo.

Para el cálculo de las limitantes climáticas se emplearon las series históricas mensuales (Periodo 19812011) de las variables: precipitación, temperatura del aire (temperatura máxima media, temperatura mínima media y temperatura media), humedad relativa y brillo solar registradas por diecisiete estaciones

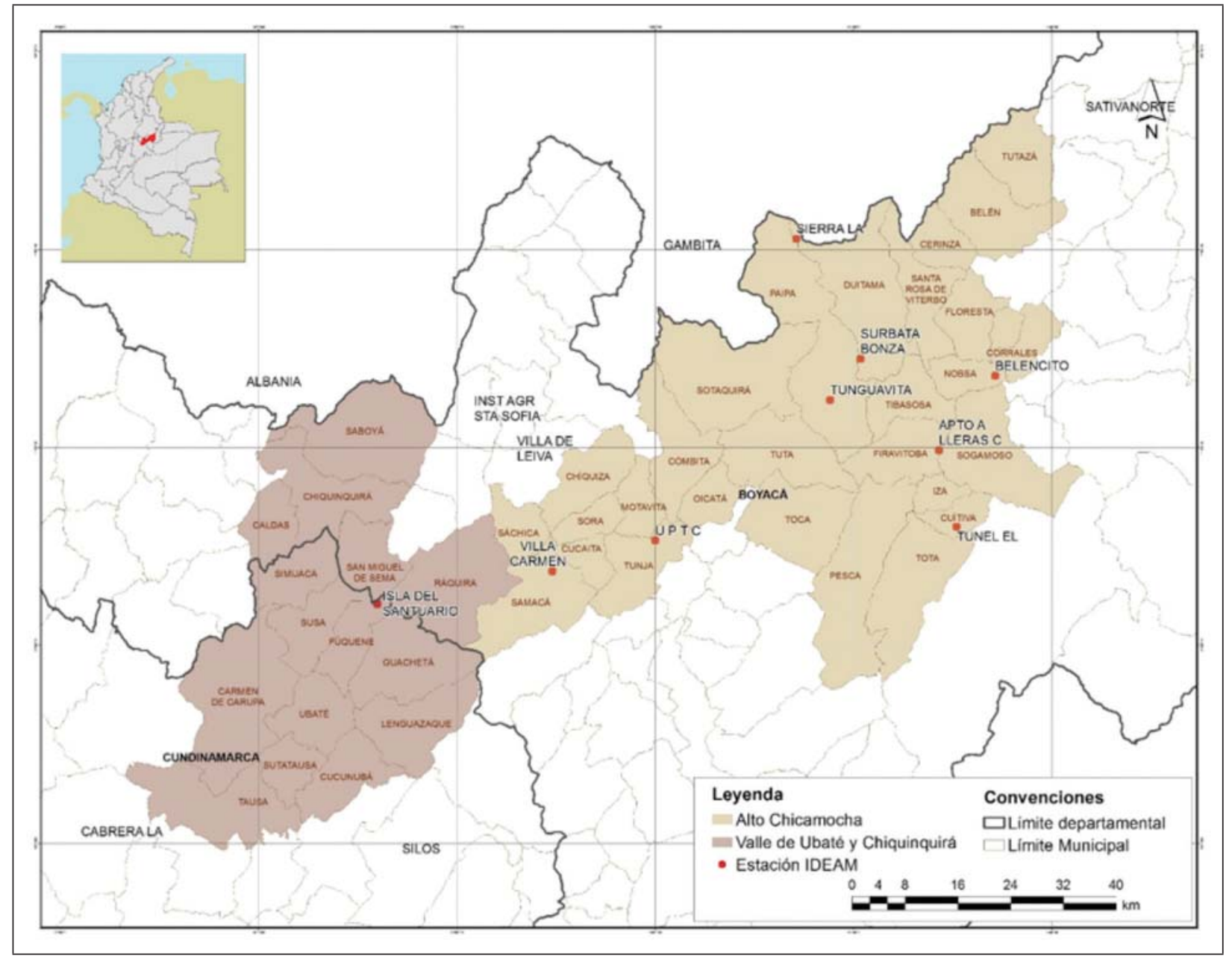

Figura 1. Valle de Ubaté y Chiquinquirá y Alto de Chicamocha, Colombia. Ubicación y coordenadas geográficas de las estaciones meteorológicas pertenecientes a la red del IDEAM empleadas en el estudio. 
pertenecientes a la red de observación meteorológica del Instituto de Hidrología, Meteorología y Estudios Ambientales (IDEAM) disponibles en el área de análisis (Fig. 1). Las series climatológicas utilizadas fueron sometidas a un proceso de calidad y complementación de datos faltantes, que incluyó etapas de verificación de la coherencia estadística y física (espacial y temporal) (Guijarro, 2014), análisis de homogeneidad de las series (Alexandersson, 1986), complementación de datos faltantes (Paulhus y Kohler, 1952; Guijarro, 2014) y validación del proceso general de control de calidad (McCuen, 1998). Se seleccionaron series de tiempo de lluvias que tuvieran máximo un 30\% de datos faltantes y para temperaturas se utilizaron series de tiempo hasta con un $40 \%$ de faltantes debido al limitado número de estaciones con registros.

Pendientes: El mapa de pendientes se elaboró a partir de un modelo digital de elevación (DEM) con resolución espacial de $30 \mathrm{~m}$, generado por la misión SRTM de la NASA. El cálculo de la pendiente se basó en la medida de la tasa de cambio de la inclinación de un píxel con relación a los vecinos (Warren et al., 2004). El mapa de pendientes fue transformado en un ráster con tamaño de píxel de $60 \mathrm{~m}$, de manera que coincidiera con los demás ráster utilizados en términos de resolución y extensión espacial.

Exposición territorial a heladas: El grado de exposición territorial a heladas se determinó a partir de la metodología descrita por Gómez-Latorre (2014). Fuentes secundarias de información de elevación, paisaje, pendiente, textura del suelo, cobertura del suelo y cercanía a cuerpos de agua se emplearon para calificar el grado exposición en cuatro categorías: ALTO, MODERADO, BAJO y NINGUNO, indicando la favorabilidad de cada variable para la ocurrencia de heladas.

Análisis de precipitación acumulada y porcentaje de días sin lluvia durante los periodos secos: Se calculó el promedio acumulado de lluvias y el porcentaje de días sin lluvias a escala mensual durante los periodos secos en la zona a partir de las series históricas diarias de precipitación para el periodo 1981 - 2011.

Índice de temperatura y humedad: Para el cálculo del Índice de Temperatura Humedad (ITH) propuesto por Thom (1959) se utilizó la calibración realizada por Valtorta y Gallardo (1996) que consideró la siguiente ecuación:

$I T H=\left(18 \mathrm{~T}_{a}+32\right)-\left(0.55-\frac{0,55 H R}{100}\right)\left(1.8 \mathrm{~T}_{a}-26\right)$
Donde $\mathrm{T}$ es la temperatura del aire $\left({ }^{\circ} \mathrm{C}\right)$ y $\mathrm{HR}$ la humedad relativa (\%). Se utilizaron las series de temperatura máxima media del aire y humedad relativa media a escala diaria para el periodo 1981 - 2011, determinando el umbral de alerta en 70 , dado que es un valor que permite generar una alerta temprana, muy cerca al valor de 72 propuesto por Johnson et al. (1961), donde se presume el inicio del estrés que afecta especialmente a las vacas de raza Bos taurus de alta producción. Se determinó la frecuencia mensual de condiciones de Alerta de ITH para cada una de las estaciones meteorológicas consideradas.

Frecuencia de ocurrencia de condiciones de déficit hídrico en el suelo: La frecuencia se calculó a partir de las series históricas mensuales del Índice de Sequía de Palmer (PDSI por sus siglas en inglés) (Palmer, 1965) calculadas para el periodo 1980 a 2011. El cálculo del índice de Palmer se generó a partir de la interpolación de los promedios mensuales de la evapotranspiración del cultivo de referencia (ETo) calculada con los datos climáticos de las estaciones de la red de IDEAM siguiendo la metodología recomendada por FAO (FAO, 2006) y los valores de Capacidad de campo (CC), punto de marchitez permanente (PMP) y punto de saturación (PS) determinados con la herramienta USDA-ARS (Hydrology and Remote Sensing Laboratory) (Saxton, 2006) a partir de las clases texturales modales, profundidad de los perfiles y horizontes modales de las unidades de suelos (IGAC, 2000; IGAC, 2004 ) en donde se ubica cada estación climatológica empleada.

El PDSI califica cualitativamente el déficit de humedad del suelo mes a mes en cinco categorías que van desde sequía incipiente hasta sequía extrema (Tab. 1). Valores del PDSI menores a $-0,5$ se consideraron como déficit hídrico. Se determinó la frecuencia mensual de condiciones de PDSI menores a $-0,5$ para los 32 años de análisis (1980 - 2011) por estación y para cada uno de los meses de los periodos secos (DEF y JJA). Con base en las frecuencias obtenidas por estación se determinaron las probabilidades mensuales de ocurrencia de condiciones de déficit hídrico para los trimestres DEF y JJA en cada una de las estaciones meteorológicas consideradas.

\section{Índice de aptitud agroclimática IAC}

El índice de aptitud agroclimática (IAC) califica el territorio en términos de su aptitud para el sistema ganadero de leche. Valores cercanos a 1 sugieren mayor aptitud (menor exposición a limitantes 
Tabla 1. Reagrupación de categorías de déficit hídrico del PDSI.

\begin{tabular}{|c|c|c|c|}
\hline Condición de humedad en el suelo & Categorías PDSI & Valor PDSI & Rango y umbrales de índice \\
\hline \multirow{3}{*}{ Déficit hídrico no extremo } & Sequía incipiente & $-0,50$ a $-0,99$ & \multirow{2}{*}{$-0,5$ y $-2,99$} \\
\cline { 2 - 3 } & Sequía reducida & $-1,99$ a $-1,00$ & \\
\cline { 2 - 3 } & Sequía moderada & $-2,99$ a $-2,00$ & $\leq-3,00$ \\
\hline \multirow{2}{*}{ Déficit hídrico extremo } & Sequía severa & $-3,99$ a $-3,00$ & $\leq-4,00$ \\
\cline { 2 - 3 }
\end{tabular}

agroclimáticas) y valores cercanos a 0 indican menor aptitud territorial para el sistema desde el punto de vista agroclimático.

Se definió una escala espacial de trabajo de 1:100.000 en la que se encuentra disponible la mayoría de la información utilizada en la obtención de las superficies de exposición a heladas y PDSI. El tamaño de píxel mínimo seleccionado fue de $60 \mathrm{~m}$, considerando que es un valor es múltiplo del tamaño de celda del DEM generado por la misión SRTM $(30 \mathrm{~m})$ y que permite generar salidas con escala espacial de 1:100.000 (Hengl, 2006).

El IAC se obtuvo mediante un análisis espacial multicriterio (Keshkamat et al., 2009) empleando el módulo "Spatial Multi-Criteria Evaluation" del software ILWIS 3.8.5. Para esto, los valores calculados para las limitantes agroclimáticas en cada estación fueron previamente interpolados a través de la distancia inversa ponderada (IDW) con una potencia 2 (Shepard, 1968). El análisis multicriterio se desarrolló en etapas que incluyeron identificación de metas y criterios, normalización y ponderación de los criterios y generación de mapas de IAC (Fig. 2).

Se normalizaron los valores de las limitantes mediante funciones valor con el fin que los criterios fueran comparables entre sí. Los valores normalizados variaron entre 0 y 1 , donde un valor de 1 representó áreas con mayor aptitud, mientras que un valor de 0 indicó áreas con menor aptitud y consecuentemente mayores limitantes para el sistema ganadero de leche. Luego de normalizar las limitantes, se les asignó el mismo peso, como una primera aproximación, para los periodos DEF y JJA (ILWIS 3.8.5) para luego vincularlos a la ponderación final que generó la distribución espacial del IAC.

\section{Análisis del IAC sobre cobertura de pastos}

La distribución espacial del IAC dentro de las zonas cubiertas con pastos fue analizada mediante la superposición de las capas espaciales de IAC para los periodos DEF y JJA y la cobertura de pastos obtenida mediante la implementación de la metodología CORINE Land Cover adaptada para Colombia a escala 1:100.000 durante el periodo 2005-2009 (IDEAM, 2012).

\section{RESULTADOS Y DISCUSIÓN}

El índice normalizado para la pendiente (Fig. 3A) indica que valores cercanos a 0 (rojo) son más limitantes para el sistema ganadero de leche debido a que la pendiente excede el $50 \%$. Hacia el norte del valle de Ubaté y Chiquinquirá se obtuvieron valores normalizados para pendiente menor a 0,2. En particular los municipios de: Tutazá, Belén, Cerinza, Santa Rosa de Viterbo, Floresta, Nobsa, Corrales, Duitama, Paipa, Combita, Sotaquirá y Chiquiza, en el departamento de Boyacá, tienen más del 25\% de su área total con pendientes mayores al 50\%. En Pesca y Tota (Boyacá), y Carmen de Carupa, Tausa, Sutatausa, Lenguazaque, Cucunubá, Susa y Simijaca (Cundinamarca), también se observó un área importante (hasta del 20\%) con valores menores a 0,2 . Los potreros de pastoreo ubicados en estas áreas son altamente susceptibles a la erosión en terracetas tipo pata de vaca, movimientos en masa y generación de cárcavas (Sadeghian et al., 2006; Murgueitio, 2003; Steinfeld, 2002; Pinzón y Amézquita 1991). La compactación resultante del tránsito de los animales afecta en forma negativa el flujo del agua a través del perfil y la estabilidad estructural, procesos que causan erosión superficial y remociones masales (Murgueitio, 2003). Esto genera reducciones en la productividad de los pastos por compactación de los suelos, ocasionando mayor dependencia de suplementos y concentrados que afectan la rentabilidad del sistema (Murgueitio, 2003). Según García (2011) la compactación y degradación del suelo en potreros con pendientes mayores al $50 \%$ ocasionan reducciones en la capacidad de carga física ( $<200 \mathrm{~d} \mathrm{ha}^{-1}$ año $^{-1}$ de unidad animal (dUA) en 


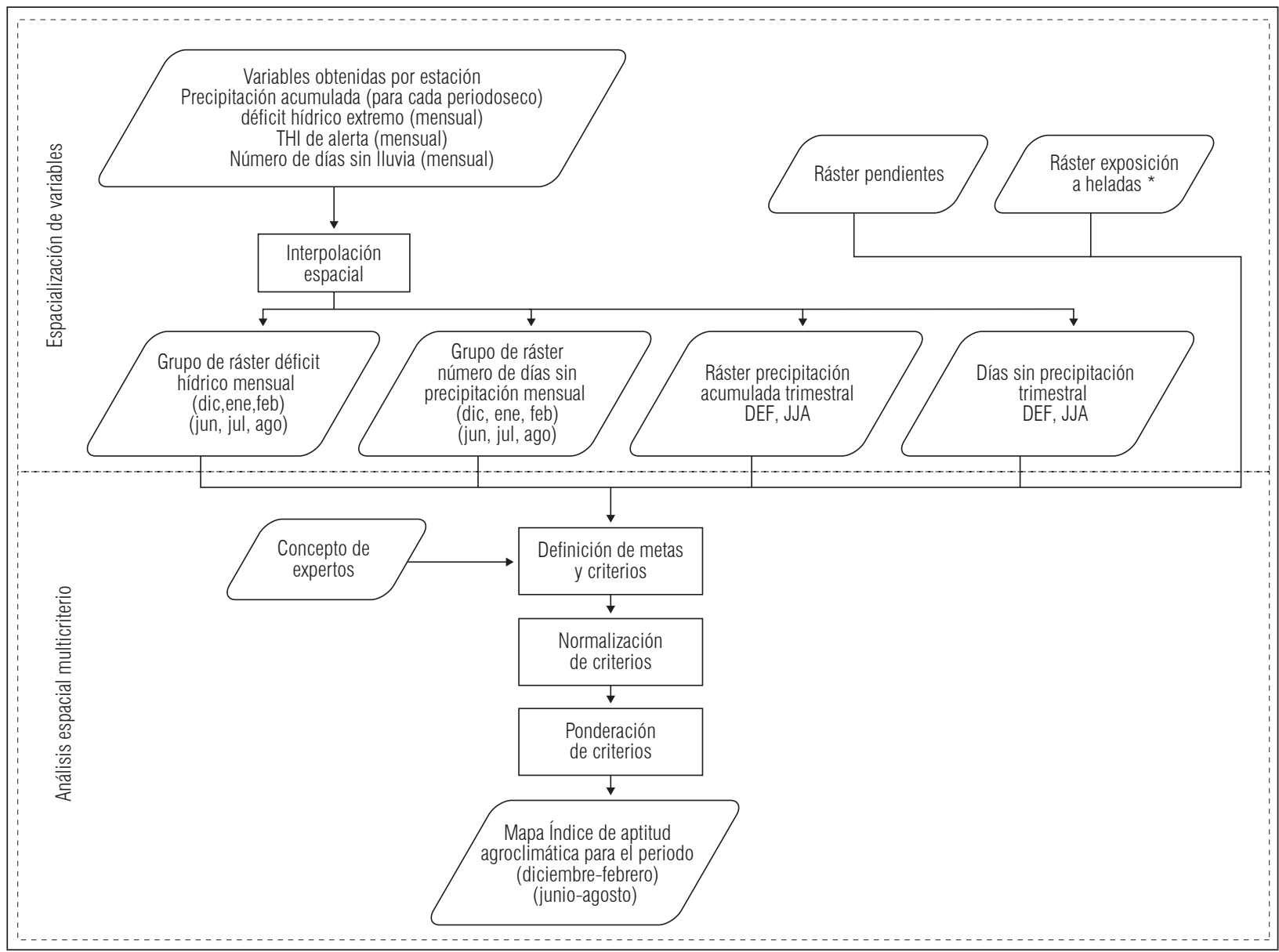

Figura 2. Diagrama de flujo del análisis espacial multicriterio para la obtención del IAC durante los periodos DEF y JJA. El criterio referente a exposición a heladas sólo se consideró en el periodo seco de DEF (* raster normalizado).

comparación con pendientes menores al 30\% donde los potreros pueden sostener una mayor carga animal acumulada (900 a 1.900 ha $^{-1}$ año-1 dUA).

El mapa de exposición territorial a heladas mostró que la mayor parte del territorio presenta alta exposición a heladas durante el trimestre DEF. Los municipios Tutazá, Belén, Cerinza, Sotaquirá, Santa Rosa de Viterbo, Sogamoso, Tota, Toca, Pesca, Combita, Chiquiza, Motavita, Tunja, Samacá, Susa, Carmen de Carupa, Tausa, Lenguazaque y Guachetá presentan hasta un $30 \%$ de su área con valores cercanos a cero (tonos rojos) y pueden ser considerados como críticos para el sistema ganadero de leche en términos de su exposición a heladas. En contraste, Sáchica, Ráquira, Cucaita, norte de Samacá, nororiente de Tunja, valles limítrofes entre Sogamoso, Tibasosa y Nobsa, norte de Fúquene, centro de Guachetá presentan un valor normalizado mayor a 0,6 indicando una menor exposición (Fig. 3b). En general el valle de Ubaté y
Chiquinquirá y la región del Alto de Chicamocha son áreas susceptibles a heladas debido a la pérdida de radiación terrestre en las horas de la noche y madrugada, las características de uso y cobertura de los suelos, su rango altitudinal (mayor a $2.500 \mathrm{msnm}$ ) y las temperaturas medias entre 9 y $12^{\circ} \mathrm{C}$ (Gómez-Latorre, 2014; Mayorga et al., 2008).

En los municipios identificados como críticos, el impacto de las heladas se relaciona principalmente con daños que pueden presentarse en el pasto kikuyo (Pennisetum clandestinum), especie forrajera predominante en la zona que presenta sensibilidad extrema a temperaturas menores de $0^{\circ} \mathrm{C}$ (Marquinez-Casas, 2001). La mayor exposición a heladas también se atribuye a la lejanía a cuerpos de agua, paisajes de planicies, valles y áreas planas a ligeramente onduladas (0-7\%) conformadas por colinas y lomas, suelos de textura gruesa y con poca capacidad de retención de agua, coberturas de páramo y subpáramo, pastos limpios, 


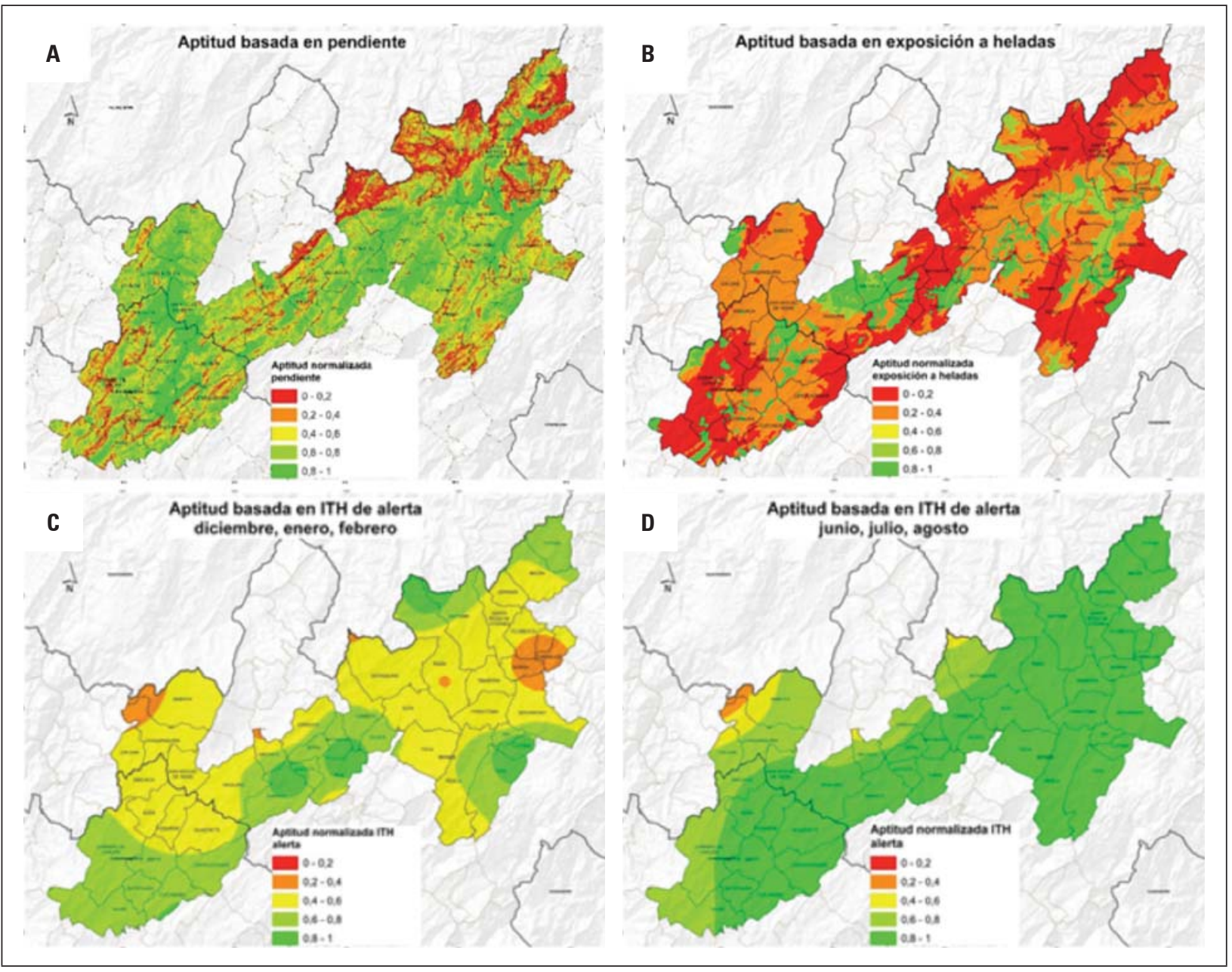

Figura 3. Distribución espacial de las limitantes agroclimáticas (escala 1:100.000) pendiente (A); Exposición a heladas (B); Probabilidad de ocurrencia de condiciones de ITH de alerta para DEF (C); Probabilidad de ocurrencia de condiciones de ITH de alerta para JJA (D), en el valle de Ubaté y Chiquinquirá y Alto de Chicamocha.

pastos naturales y sabanas, mosaico de pastos y cultivos, pastos enmalezados o enrastrojados (Gómez-Latorre, 2014).

La normalización del ITH sugiere que valores cercanos a 0 indican una mayor frecuencia de ocurrencia de alertas de estrés térmico para Bos taurus. Durante DEF la mayor parte del área de estudio presentó valores normalizados de alerta por estrés térmico (ITH>71) menores a 0,6 , indicando que en este periodo hay una mayor ocurrencia de eventos estresantes para Bos taurus en términos de temperatura y humedad. Los municipios en donde predominaron estos valores fueron Saboya y Chiquinquirá, norte de Sáchica, Villa de Leyva, región central de Paipa y en la zona oriental, en los municipios Sogamoso, Nobsa, Corrales y Floresta (Fig. 3C).
Durante la época seca JJA la mayor parte del territorio presentó un valor normalizado de alerta por estrés térmico (ITH>71) mayor a 0,8, principalmente hacia el área con influencia climática del piedemonte llanero, cuya temporada de lluvias empieza durante estos meses. Durante las dos épocas secas los valores más bajos $(<0,6)$ persisten en el occidente de Saboyá y noroccidente de Chiquinquirá y Sotaquirá (Fig. 3D). En estas áreas durante los periodos secos pueden presentarse altas temperaturas, comúnmente denominados "olas de calor" (Arias et al., 2008; Brown-Brandl, et al., 2006; Hahn et al., 1993), que implican períodos de calor y humedad estresantes para el ganado. Esto puede generar incrementos en el consumo diario de agua y detrimento en el consumo diario de materia seca como respuesta al aumento en la temperatura (Bicudo y Gates, 2002). 


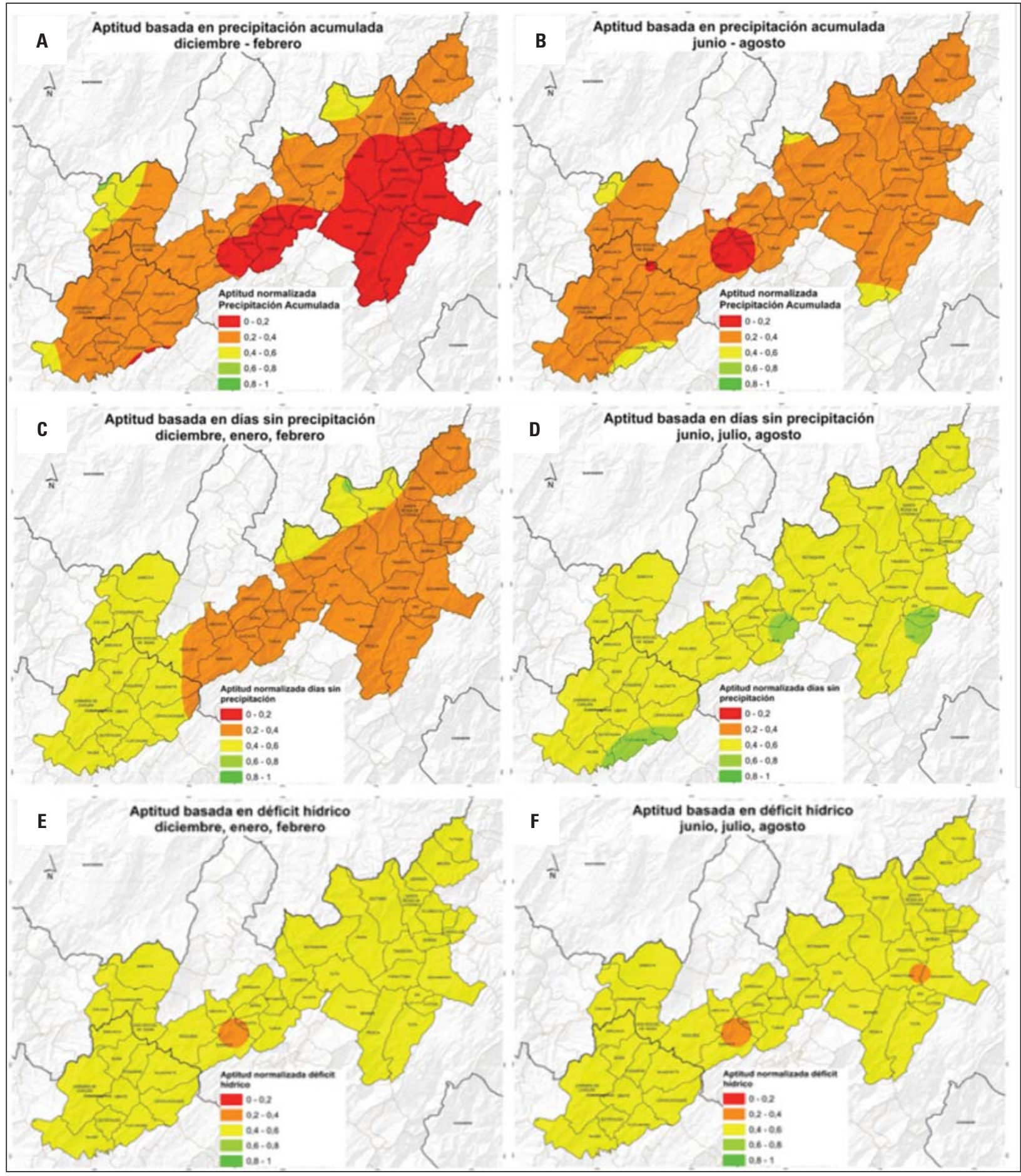

Figura 4. Distribución espacial de las limitantes agroclimáticas (escala 1:100.000) de precipitación acumulada en DEF (A); en JJA (B); Días sin precipitación en DEF (C); en JJA (D); Déficit hídrico en DEF (E); en JJA (F), en el valle de Ubaté y Chiquinquirá y Alto de Chicamocha.

Para evitar los efectos del exceso de calor, los animales provocan una reducción en su desempeño productivo debido a la activación de mecanismos termorregulatorios (fisiológicos y de comportamiento) para conservar la temperatura corporal. Así mismo, modifican su comportamiento habitual disminuyendo 
el tiempo dedicado a consumir alimento y el que permanecen echados, aumentando el tiempo dedicados a beber agua y el que permanecen de pie cerca de los bebederos (Brown-Brandl et al., 2006).

En general los valores del porcentaje normalizado de días sin precipitación acumulada en la mayor parte del territorio fueron mayores durante el periodo JJA en comparación con DEF, lo que indica una mejor distribución de lluvias durante el periodo seco de mitad de año (JJA) y posiblemente un menor impacto de las deficiencias hídricas sobre las pasturas establecidas (Fig. 4A y 4B). Pese a que en la mayor parte del área los valores normalizados de precipitación acumulada son predominantemente menores a 0,4 en los dos periodos, una mayor área con valores $<0,2$ (tonos rojos) de precipitación acumulada se observó durante DEF (Fig. 4C y 4D). En esta área, correspondiente los municipios Iza, Cuitiva, norte de Tota, oriente de Firavitova y suroeste de Sogamoso, noroeste de Tunja y suroriente de Motavita; los volúmenes de precipitación deficientes pueden reducir la productividad, principalmente en fincas de ladera y donde el manejo agronómico no es el adecuado.

La producción de leche en al altiplano cundiboyacense es altamente estacional, con ciclos de alta producción en invierno y baja producción en verano, determinada principalmente por los volúmenes de lluvias que determinan la disponibilidad de alimentación para los animales (Arreaza, et al., 2012; Arreaza, 2012; Cardozo et al., 2008). Teniendo en cuenta esto, se puede afirmar que se cuenta con mayor disponibilidad de lluvias y mejor distribuidas durante el periodo seco de mitad de año JJA que durante el periodo DEF.

La frecuencia normalizada de déficit hídrico y su distribución espacial fue similar en ambos periodos secos. Predominaron valores entre 0,4 y 0,6 , salvo en los municipios de Samacá, Cucaita, Sogamoso y Firavitova en donde los valores entre 0,2 y 0,4 (Figura $4 \mathrm{e}$ y $4 \mathrm{f}$ ) son un indicativo de mayores limitaciones debidas a déficit hídrico en el suelo. Bajo esta condición hay una mayor presión de plagas reportadas en la zona como el chinche de los pastos (Collaria scenica y Collaria columbiensis) (Bernal, 2009; Castro et al., 2009) y reducciones del valor nutritivo, la disponibilidad, calidad y digestibilidad de forraje (Cuesta et al., 2005) generadas a causa de menores tasas de crecimiento y mayores períodos de descanso de las pasturas (entre 40 y $90 \mathrm{~d}$ ). Este cambio en las tasas de crecimiento afecta directamente la calidad del forraje, debido a la movilización de nutrientes del follaje hacia la inflorescencia y lignificación de los tallos florales (Bernal, 1994; Huang, 1999). Por lo anterior hay un menor consumo de forraje por parte del animal y finalmente una menor productividad por cabeza.

\section{IAC}

En general durante el periodo DEF el 33\% (192.369 ha) del área total del valle de Ubaté y Chiquinquira y Alto de Chicamocha presentó valores bajos de IAC (menores a 0,4). En particular en el departamento de Boyacá los valores más bajos están en áreas de los municipios Busbanzá, Sogamoso, Corrales, Tutazá, Belén, Santa Rosa de Viterbo, Floresta, Nobsa, Sotaquirá, Cerinza, Pesca y Chíquiza, los cuales presentaron entre el 50 y $75 \%$ de su área con valores de IAC $<0,4$ (Fig. 5A). Durante el trimestre JJA solo el 1,5\% presento valores de IAC menores a 0,4 (31,5\% menos que en el periodo DEF) y el municipio de Chíquiza permanece con buena parte de su superficie $(22 \%$ del área total) con valores que indican baja aptitud agroclimática (IAC <0,2) (Fig. 5B). La menor aptitud, en términos de superficie con valores bajos del IAC observada durante el trimestre DEF es debida a una menor acumulación de precipitación, mayor frecuencia de alerta (ITH >71) para Bos taurus y alta exposición a heladas en esta temporada.

Los valores más altos del IAC $(0,6-0,8)$ se encontraron en menos del 3,5\% (17.045 ha) durante DEF (en los municipios Fúquene, Cuitiva, Táusa y Chiquinquirá) y en el 12\% (64.549 ha) del área en JJA (municipios Oicata, Cucunubá, Cuitiva, Ubaté, Tota, Tunja, Iza, Motavita, Lenguazaque, Motavita, Toca Tibasosa, Tuta, Sutatausa, Tausa, Firavitova, Duitama y Pesca). En estas áreas hay una mayor aptitud agroclimática para el sistema ganadero de leche durante las temporadas de más bajos volúmenes de precipitación (Fig. 5).

\section{Análisis del IAC y coberturas de pastos en el área de análisis}

Durante el trimestre DEF, el 24\% del área con cobertura de pastos presentó valores del IAC $<0,4$, mientras que para el periodo JJA solo en el 0,5\% del área de pastos se observó esta condición (Fig. 6). Esto indica que durante el periodo DEF hay mayor exposición de las coberturas de pastos a condiciones agroclimáticas limitantes (83.408 ha). En estas áreas de valores bajos de IAC, el desarrollo y productividad están relacionados con la composición de la pradera, pues 


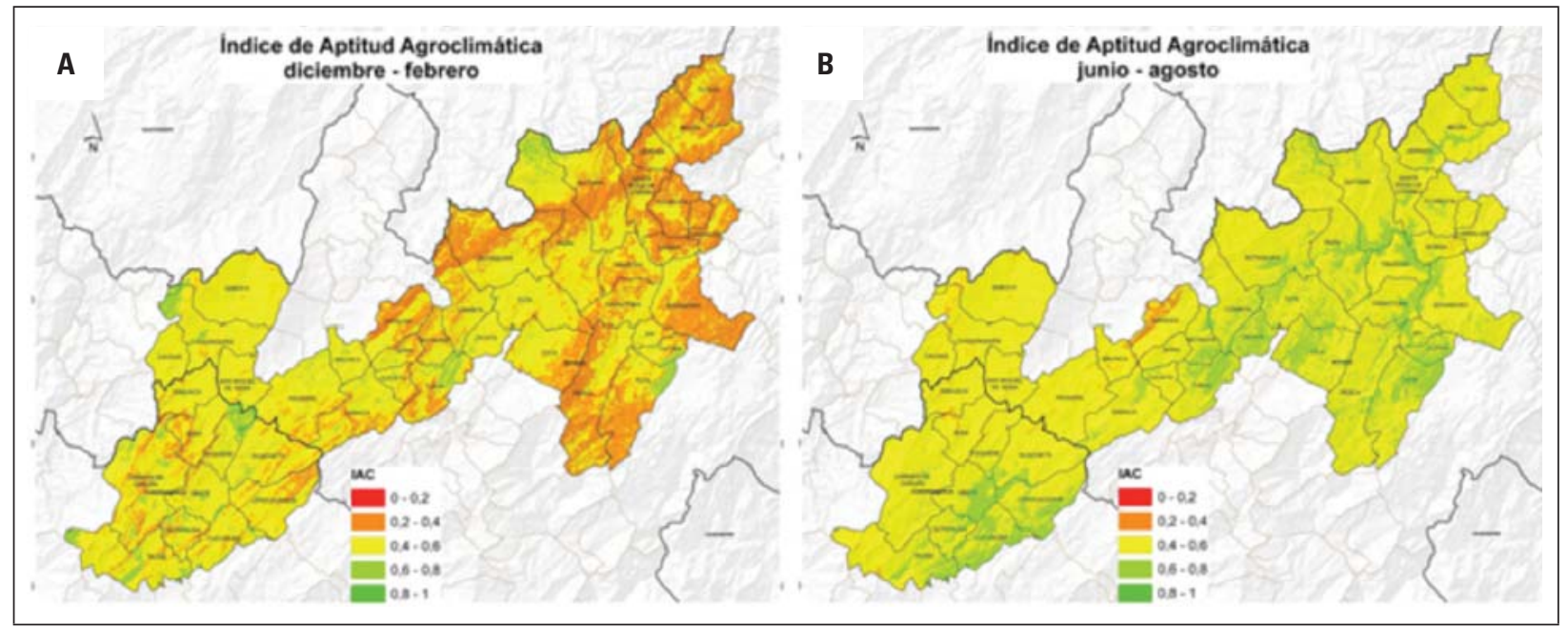

Figura 5. Distribución espacial del IAC para los periodos secos DEF (A) y JJA (B).

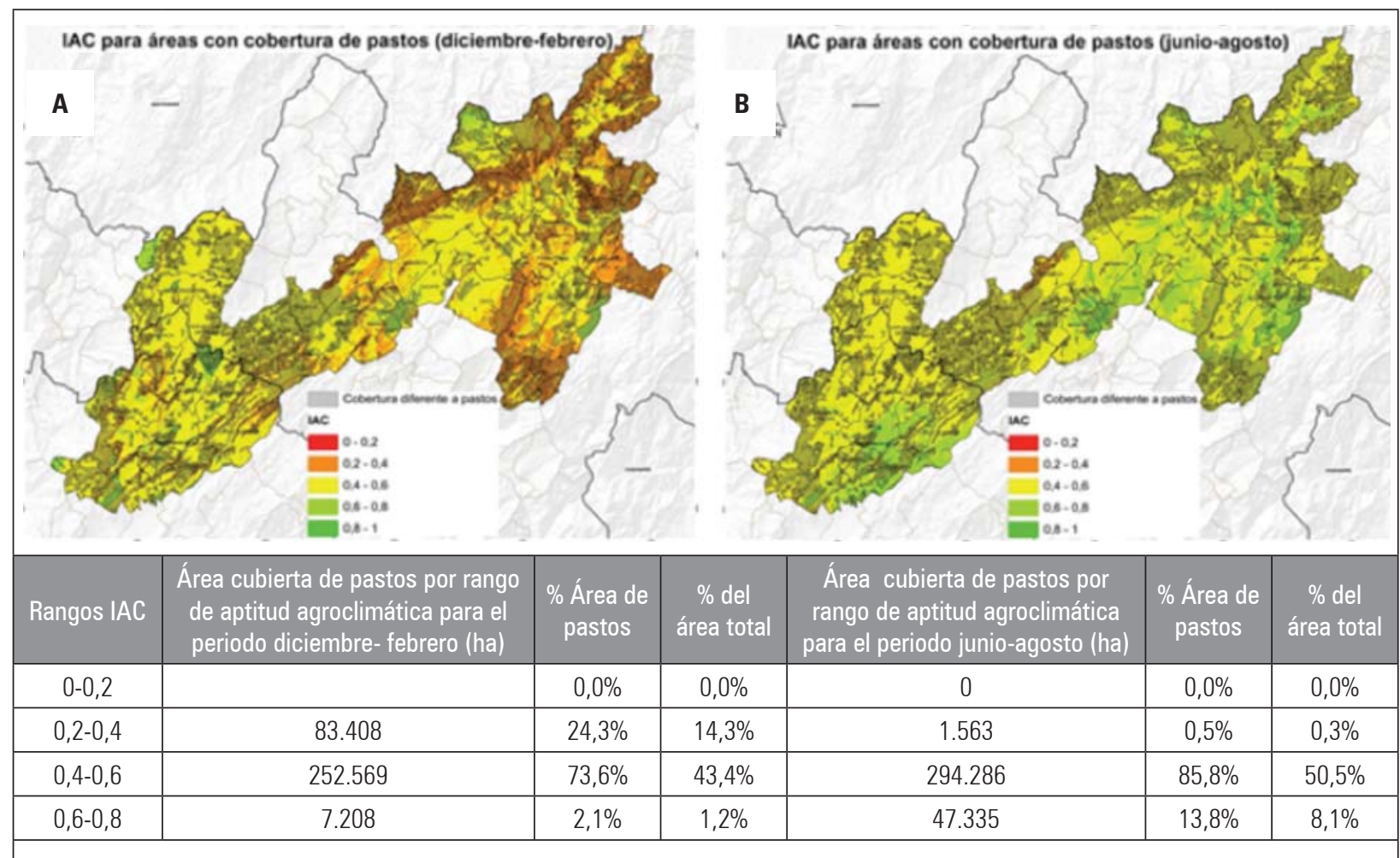

Figura 6. Distribución espacial de IAC (escala 1:100.000) en áreas (tabla) con cobertura de pastos para los periodos secos DEF (A) y JJA (B).

cada especie de pasto tiene diferencias en términos de sensibilidad o tolerancia a eventos climáticos limitantes. El pasto kikuyo (Pennisetum clandestinum), recientemente rebautizado como Cenchrus clandestinus Hochst. ex Chiov., es el material predominante en el área de estudio además de ser el más tolerante a la sequía, pero es altamente susceptible a las heladas
(Carulla y Ortega, 2016; Bernal, 2009; Castro et al., 2009). Los raigrases (Lolium spp.) aunque presentan mayores rendimientos en altitudes entre 2.000 y $2.800 \mathrm{msnm}$, son materiales resistentes a heladas y toleran el ataque del chinche de los pastos (Carulla et al., 2004), que es más fuerte durante épocas secas (Restrepo y Escobar, 2007). Por su parte la falsa Poa 
(Holcus lanatus), que crece abiertamente en praderas naturales y en mezcla con otros cultivos forrajeros, tolera sequías y heladas, pero es altamente susceptible al chinche de los pastos (Collaria spp.) (Restrepo y Escobar, 2007).

Durante el trimestre JJA (Fig. 6B), además de una menor exposición de la cobertura de pastos a limitantes agroclimáticas, cerca de 47.000 ha de pastos están en áreas con la más alta aptitud agroclimática (valores IAC entre 0,6 y 0,8$)$.

\section{CONCLUSIONES}

Los municipios que presentaron más baja aptitud agroclimática en términos de las variables estudiadas (pendiente, heladas, alerta de estrés térmico, lluvias y déficit hídrico en el suelo) y el IAC para el sistema de ganadería de leche en el valle de Ubaté y Chiquinquirá y Alto de Chicamocha fueron Sogamoso, Corrales, Tutazá, Belén, Santa Rosa de Viterbo, Floresta, Nobsa, Sotaquirá, Cerinza, Pesca, Chíquiza, Tausa, Samacá, Cucaita, Sogamoso y Firavitova.

A partir de los resultados del estudio es posible concluir que las praderas y en general el sistema de ganadería de leche del valle de Ubaté y Chiquinquirá y el Alto de Chicamocha presentan una mayor exposición a limitantes agroclimáticas durante el trimestre DEF que durante el trimestre JJA

Con respecto al IAC, pese a que cada variable influyó de forma determinante, la pendiente presentó una condición crítica para la producción de biomasa de la pradera en términos del impacto sobre las demás variables como el déficit hídrico y la exposición a heladas. En áreas de pendientes pronunciadas hay mayor expresión de condiciones de déficit hídrico en el suelo $\mathrm{y}$ de heladas principalmente en colinas y lomas con formación alongada de sus partes más altas.

Los resultados contribuyen a la identificación de áreas en donde el sistema de ganadería de leche podría ser más afectado cuando hay periodos secos debidos a eventos de variabilidad climática asociados y no asociados a ENSO. Esto permite definir áreas críticas sobre las cuales se deben establecer medidas preventivas y de adaptación a la variabilidad climática, como la organización del recurso técnico, infraestructura de riego, la selección del germoplasma para establecimiento de praderas de acuerdo con las principales limitantes agroclimáticas entre otras.

\section{REFERENCIAS BIBLIOGRÁFICAS}

Alexandersson, H. 1986. A homogeneity test applied to precipitation data. J.Climatol. 6, 661-675. Doi: 10.1002/ joc. 3370060607

ANALAC. 2012. Balance de la leche en Colombia año 2011. Asociación Nacional de Productores de Leche, Bogotá, Colombia.

Arias, R.A, T.L. Mader y P.C. Escobar. 2008. Factores climáticos que afectan el desempeño productivo del ganado bovino de carne y leche. Arch. Med. Vet. 40, 7-22.

Arreaza, L. C., G. Amado, C. Londoño, D. Ballesteros y J. Herrera. 2012. Recomendaciones para la fabricación de ensilajes con cereales en climas fríos. Corporación Colombiana de Investigación Agropecuaria, Bogotá, Colombia.

Arreaza, L.C. 2012. Recomendaciones generales de alimentación en épocas de crisis. Corporación Colombiana de Investigación Agropecuaria, Bogotá, Colombia.

Bernal, J. 1994. Pastos y forrajes tropicales. $3^{a}$ ed. Banco Ganadero, Bogotá, Colombia.

Bernal, J. 2009. Pastos mejorados. En: Pastos y forrajes; http://hasp.axesnet.com/contenido/documentos/ Friocapitulo7.pdf; consulta: junio de 2014.

Bicudo J. y R. Gates. 2002. Water consumption, air and water temperature issues related to portable water systems for grazing cattle. En: ASAE, Annual International Meeting/CIGR XVth World Congress . Chicago, IL, USA.

Brown-Brandl, T.M., J.A. Nienaber, R.A. Eigenberg, T.L. Mader, J.L. Morrow y J.W. Dailey. 2006. Comparison of heat tolerance of feedlot heifers of different breeds. Livest. Sci. 105, 19-26. Doi: 10.1016/j.livsci.2006.04.012

Brunini, O., J.P. Carvalho, A. P.C. Brunini, A. L. Padua-Junior, S.F. Adami y P.L.G. Abramides. 2010. Agroclimatic zoning and climatic risks for sugarcane in Mexico: a preliminary study considering climate change scenarios. Proc Int. Soc. Sugar Cane Technol. 27, 1-13.

Brunini, O., A.L. Pádua-Junior, J.P. Carvalho, H. Prado, A. Brunini, R. Viegas y R. Campanha. 2009. Zoneamento agroambiental da cultura da cana-de-açúcar no Estado de São Paulo, 2nd versión. Secretearia de Agricultura e Abastecimento, Edo. de Sao Paulo, Brasil.

Cardozo, F. S., L. Sánchez, I. Baquero, M. Ramirez, R. Díaz y E. Villaneda. 2008. Uso óptimo de insumos y modernas herramientas de costeo: estrategias para la competitividad de la lechería especializada del trópico alto colombiano. Corporación Colombiana de Investigación Agropecuaria, Bogotá, Colombia.

Carulla, J.y E. Ortega. 2016. Sistemas de producción lechera en Colombia: retos y oportunidades.. Arch. Latinoam. Prod. Animal 24(2), 83-87. 
Carulla, J., E. Cárdenas, N. Sánchez y C. Riveros. 2004. Valor nutricional de los forrajes más usados en los sistemas de producción lechera especializada de la zona andina colombiana. (Colombia). p. 21-38. En: Seminario Nacional de Lechería Especializada: Bases Nutricionales y su Impacto en la Productividad, Medellín, Colombia.

Castro, E., J.E. Mojica, J. León, M. Pabon, J. Carulla y E. Cardenas. 2009. Balance de nitrogeno en pastura de gramínea más Lotus uliginosus en la sabana de Bogotá, Colombia. Rev Cienc. Tecnol. Agropecu. 10(1), 91101. Doi: 10.21930/rcta.vol10-num1

CEPAL. 2015. Estudio económico de América Latina y el Caribe, 2015 (LC/G.2645-P). Comisión Económica para América Latina y el Caribe. Santiago, Chile.

Corpoica. 2004. Informe técnico final: Caracterización de los sistemas de producción de leche del trópico de altura de los departamentos de Boyacá y Cundinamarca. Corporación Colombiana de Investigación Agropecuaria, sede C.I. Tibaitatá, Mosquera, Colombia.

Cuesta M.P., E.H. Mateus, M.O. Santana y J.B. Henriquez. 2005. Producción y utilización de recursos forrajerosen sistemas de producción bovina de las regiones Caribe y valles interandinos. Arteprint, Bogota, Colombia.

DANE. 2016. Encuesta nacional agropecuaria ENA 2015 Boletín técnico. Departamento Administrativo Nacional de Estadística, Bogotá, Colombia.

Díaz, J. y J. López. 2000. Evaluación del potencial para acuacultura costera de camarón en el entorno de la laguna de Mar Muerto, mediante la aplicación de técnicas de análisis multicriterio con un SIG. Invest. Geo. 41, 62-80.

FAO. 2006. Evapotranspiración del cultivo. Estudio FAO riego y drenaje $\mathrm{N}^{\circ}$ 56. FAO, Roma, Italia.

FAO. 1997. La agricultura y los cambios climáticos: la función de la FAO. FAO Noticias. Roma, Italia.

García, E. D. 2011. Evaluación del impacto del uso ganadero sobre suelo y vegetación en el Sistema Agroforestal Quesungual (SAQ) en el sur de Lempira, Honduras. Tesis de maestría. Centro Agronómico Tropical de Investigación y Enseñanza (CATIE). Turrialba, Costa Rica.

Guijarro, J. A. 2014. User's guide to climatol. Agencia Estatal de Meteorología (AEMET), Madrid, España.

Gómez-Latorre, D. 2014. Caracterización, pronóstico y alternativas de manejo de las heladas en el sistema de producción lechero del Valle de Ubaté y Chiquinquirá (Colombia). Tesis de maestría. Facultad de Ciencias, Universidad Nacional de Colombia, Bogotá, Colombia.

Hahn, G.L., J.A. Nienaber y R.A. Eigenberg. 1993. Environmental influences on the dynamics of thermoregulation and feeding behavior in cattle and swine. pp. 1106-116. En: $4^{\text {th }}$ Int. Symp. Livestock Environment IV. University of Warwick, Amer. Soc. Agric. Eng., Coventry, UK.
Hengl, T. 2006. Finding the right pixel size. Comp. Geosci. 32(9), 1283-1298, Doi: 10.1016/j.cageo.2005.11.008

Holmann, F., P. Argel, L. Rivas, D. White, R. D. Estrada, C. Burgos, E. Perez, G. Ramirez y A. Medina. 2004. Benefits and costs of rehabilitation of degraded pastures in Honduras. Pasturas Trop. 26(3), 13-28,

Huang, B. 1999. Water relations and root activities of $\mathrm{Bu}$ chloe dactyloides and Zoysia japonica in response to localized soil drying. Plant Soil 208, 179-186. Doi: 10.1023/A:1004472314631

IDEAM. 2012. Mapa de coberturas de la tierra. Metodología Corine Land Cover adaptada para Colombia escala 1:100.000, periodo 2005-2009. Instituto de Hidrología, Meteorología y Estudios Ambientales, Bogotá, Colombia.

IGAC. 2005. Estudio general de suelos y zonificación de tierras de departamento de Boyacá. IGAC, Bogotá.

IGAC. 2000. Estudio general de suelos y zonificación de tierras de departamento de Cundinamarca. IGAC, Bogotá.

IPCC. 2012. Managing the risks of extreme events and disasters to advance climate change adaptation. Cambridge University Press. Cambridge.

Johnson, H.D., H.H. Kibler, A.C. Ragsdale, I.L Berry y M.D. Shanklin. 1961. Role of heat tolerance and production level in responses of lactating Holsteins to various temperature-humidity conditions. J. Dairy Science. 44, 1191. Doi: 10.3168/jds.S0022-0302(61)89869-X

Keshkamat, S., J. Looijen y M. Zuidgeest. 2009. The formulation and evaluation of transport route planning alternatives: a spatial decision support system for the Via Baltica project, Poland. J. Transp. Geogr. 17, 54-64. Doi: 10.1016/j.jtrangeo.2008.04.010

Marquinez-Casas, X. 2001. Determinación de posibles mecanismos fisiológicos de tolerancia a heladas en los pastos kikuyo y falsa poa. Tesis de maestría. Facultad de Agronomía, Universidad Nacional de Colombia. Bogotá.

Mayorga, R., Y. González, y G. Hurtado, 2008. Las heladas en Colombia. Documento técnico de respaldo a la información en la página web del IDEAM. Instituto de Hidrología, Meteorología y Estudios Ambientales, Bogotá, Colombia.

McCuen, R. 1998. Hydrologic analysis and design . Prentice-Hall, Englewood Cliffs, NJ, USA.

Moeletsi, M.E. and S. Walker. 2012. Assessment of agricultural drought using a simple water balance model in the Free State Province of South Africa. Theor. Appl. Climatol. 108, 425-450. Doi: 10.1007/s00704-011-0540-7

Murgueitio, E. 2003. Impacto ambiental de la ganadería de leche en Colombia y alternativas de solución. Livest. Res. Rural Dev. 15(10). 
Palmer,W.1965. MeteorologicaldroughtRes. PaperNo.45.Department of Commerce, Weather Bureau, Washington DC, USA.

Paulhus, J. y M. Kohler. 1952. Interpolation of missing precipitation records. Month. Weath. Rev. 80, 129-133. Doi: 10.1175/1520-0493(1952)

Pinzón, A. y E. Amézquita. 1991. Compactación por ganadería intensiva en algunos suelos del Caquetá (Colombia). Suelos Ecuat. 21(1), 104-111.

Restrepo, J.S. y A.F. Escobar. 2007. Evaluación de algunos parámetros fisiologicos del raygrass bestfor (Lolium perenne), bajo condiciones de estres hídrico. Trabajo de grado. Facultad de Medicina Veterinaria y Zootecnia, Corporación para Estudios en la Salud (Universidad CES), Medellín, Colombia.

Sadeghian, S., M. Mejía y P. Arcila. 2006. Composición elemental de frutos de café y extracción de nutrientes por la cosecha en la zona cafetera de Colombia. Rev. Cenicafé 57(4), 251-261.

Sánchez, L. y E. Villaneda. 2009. Renovación y manejo de praderas en sistemas de producción de leche especializada en el trópico alto colombiano. Corpoica, Mosquera, Colombia.

Saxton, K.E. y W.J. Rawls . 2006. soil water characteristic estimates by texture and organic matter for hydrologic solutions. Soil Sci. Soc. Am. J. 70, 1569-1578, Doi: 10.2136/sssaj2005.0117

Sivakumar, V., W.C. Tan, E.J. Murray y J. McKinley. 2006. Wetting, drying and compression characteristics of compacted clay. Geotechn. 56(1-1), 57-62. Doi: 10.1680/geot.2006.56.1.57
Shepard, D. 1968. A two-dimensional interpolation function for irregularly-spaced data. pp. 517-524. En: Proceedings of the $196823^{\text {th }}$ ACM National Conference. Association for Computing Machinery, New York, USA.

Steinfeld, H. 2002. Ganadería y medio ambiente en Centroamérica. Intensificación de la ganadería en Centroamérica; En: FAO, Roma, Italia.

Tapasco, J., M. Quintero, N. Uribe, J. Valencia, G. Romero, S. Calderón, D. A. Ordóñez, A. Álvarez, L. Sánchez-Aragón y C. E. Ludeña. 2015. Impactos económicos del cambio climático en Colombia: Recurso hídrico. Banco Interamericano de Desarrollo, Monografía No. 258, Washington D.C., USA.

Thom, E.C. 1959. The discomfort index. Weatherwise 12, 57-59, Doi: 10.1080/00431672.1959.9926960

Valtorta, S. E. y M.R. Gallardo. 1996. El estrés por calor en producción lechera. Instituto Nacional de Tecnología Agropecuaria INTA, Estación Experimenta Rafaela, Argentina. Miscelánea 81, 173-185.

Warren, S.D., M.G. Hohmann, K. Auerswald y H. Mitasova. 2004. An evaluation of methods to determine slope using digital elevation data. Catena 58, 215-233, Doi: 10.1016/j.catena.2004.05.001

Yazdanpanah, H., Khalili-A, Hajam-S, Khamali-Gy A. Vezvai. 2001. Agroclimatic zoning of Azarbayjan-Sharghi province for rainfed almond using GIS. En: Geospatial World; https://www.geospatialworld.net/article/ agroclimatic-zoning-of-azarbayjan-sharghi-province-for-rainfed-almond-using-gis/, consulta: enero de 2017. 